\section{Migrationsverhalten des Schaftes im Zementmantel}

Fälschlicherweise wird häufig angenommen, dass sich die Position einer Prothese in einem Zementmantel nicht verändern kann. Eine RSA-Analyse des polierten C-Schaftes zeigte bereits eine distale Migration nach einem 2-Jahres-Follow-up. Diese RSA-Studie von von Schewelov et. al. zeigt ein weiteres Migrationsverhalten mit einer kontinuierlichen distalen Migration und Innenrotation des Schaftes im Zementmantel.

von Schewelov T et al. Continuous distal migration and internal rotation of the C-stem prosthesis without any adverse clinical effects - a RSA study of 33 primary total hip arthroplasties followed for up to ten years. Bone Joint J 2014; 96: 604-608

\section{Einleitung}

Aufgrund der bekannten Nachteile im Revisionsfall ist in Deutschland der Anteil zementierter Hüfttotalendoprothetik gering. Umso wichtiger ist es, das Verhalten dieser zementierten Implantate zu kennen und richtig zu interpretieren, um unnötige Indikationen zum Hüft-TEP-Wechsel zu vermeiden. Bei der zementierten Implantation verankert sich die Prothese über einen Knochenzementmantel in der Spongiosa des Knochens. Bei polierten 3-fach-konischen Schäften besteht ein Kriecheffekt zwischen Schaft und Knochenzementmantel. Somit kann es über die Jahre nach Implantation eines zementierten Schaftes zur Veränderung der Position des Schaftes im Zementmantel kommen. Eine Radio-Stereometrie-Analyse (RSA) des polierten C-Schaftes (DePuy International, Leeds, UK) zeigte bereits eine distale Migration nach einem 2-Jahres-Follow-up. Unklar war, ob und inwieweit eine weitere Migration über diesen Follow-up hinaus nachweisbar ist, sodass sich die vorliegende Studie sich der Thematik annahm.

\section{Methodik}

$\nabla$

2002 erfolgte bei 36 Patienten die Implantation einer Hüft-TEP unter Verwendung eines zementierten C-Schaftes sowie eines zementierten PE-Inlays. Nach 2 und 5 Jahren konnten noch 33 Patienten, nach 7 Jahren noch 30 Patienten und nach 10 Jahren noch 25 Patienten zur Nachuntersu-
Die Studie bestätigt: Auch zementierte Hüftprothesen zeigen ein Migrationsverhalten (Bildquelle: istockphoto). kontinuierliche distale Migration des Schaftes über den gesamten Nachuntersuchungszeitraum von durchschnittlich $2 \mathrm{~mm}$ (0,5 bis $4,0 \mathrm{~mm})$. Zusätzlich konnte eine Rotation des Schaftes nach innen aufgezeigt werden $\left(1,6^{\circ}\right.$ außen bis $6,6^{\circ}$ innen).

\section{Kommentar \\ $\nabla$}

Die Ergebnisse dieser RSA-Studie bestätigen das Migrationsverhalten, wie es bereits durch andere Studien anhand des zementierten Exeterschaftes gezeigt werden konnte. Für den Klinikalltag ist es essentiell zu berücksichtigen, dass es bei zementierten Hüft-TEPs nach Jahren noch zu einer Migration kommen kann. Diese Studie liefert sehr interessante Fakten insbesondere für Orthopäden und Unfallchirurgen in Deutschland, wo der Prozentsatz der zementfrei fixierten Hüft-TEPs dem der zementierten Hüft-TEPs deutlich überlegen ist und somit die Souveränität bei der Beurteilung zementierter Schäfte geringer sind.

Fälschlicherweise wird angenommen, dass sich eine Prothese in einem Zementmantel nicht bewegen könne. Diese Studie zeigt ein geringes kontinuierliches Migrationsverhalten einer Prothese im Zementmantel, das auch über einen Zeitraum von 10 Jahren anhalten kann. Die Methodik dieser Arbeit ist sehr gut. Die RSA-Analyse stellt den Goldstandard zur Evaluation des Migrationsverhaltens dar. Eine RSA-Kontrolluntersuchung bewies eine hohe Genauigkeit dieser Technik. Das Follow-up von 10 Jahren ist außergewöhnlich und stellt neue Erkenntnisse dar. Einschränkend ist zu nennen, dass von den initial 36 Hüft-TEP-Patienten bei der 10-JahresRSA-Analyse nur noch 14 Patienten eingeschlossen werden konnten. Dies ist allerdings darin begründet, dass über die Jahre einige Tantalkügelchen, die für die RSAAnalyse in das proximalen Femur eingebracht worden sind, unbeständig wurden und somit eine RSA-Analyse nicht mehr möglich war.

\section{Dr. med. Thilo Flörkemeier}

Orthopädie der Medizinischen Hochschule Hannover (MHH) im Annastift Thilo.Floerkemeier@ddh-gruppe.de 